\section{Radiofrequency ablation of recurrent thyroid cancers: anatomy-based management}

\author{
Min Kyoung Lee ${ }^{1,2}$, Jung Hwan Baek', Sae Rom Chung ${ }^{1}$, Young Jun Choi', \\ Jeong Hyun Lee', So Lyung Jung ${ }^{2}$
}

'Department of Radiology and Research Institute of Radiology, Asan Medical Center, University of Ulsan College of Medicine, Seoul; ${ }^{2}$ Department of Radiology, Yeouido St. Mary's Hospital, College of Medicine, The Catholic University of Korea, Seoul, Korea

Although differentiated thyroid cancer has an excellent prognosis and low mortality, its recurrence rate has been reported to be very high. While surgery is recommended as the standard treatment for recurrent thyroid cancer, reoperation may increase the incidence of complications due to fibrosis and anatomical distortion. In patients with high surgical risk or those who refuse repeated surgery, ultrasonography-guided ablation techniques including radiofrequency ablation, ethanol ablation, and laser ablation are suggested as alternative treatments. In this manuscript, we introduce an anatomy-based management concept for radiofrequency ablation for recurrent thyroid cancer, and present a review of the relevant literature, including indications, basic techniques, clinical outcomes, and complications.

Keywords: Thyroid neoplasms; Ultrasound; Radiofrequency ablation; Recurrence

Key points: Ultrasonography-guided radiofrequency ablation is an alternative treatment for recurrent thyroid cancer, with good outcomes and low complication rates.

\section{Introduction}

Differentiated thyroid cancers (DTCs), including papillary and follicular cancers, are the most common subtype of thyroid malignancies, representing more than $90 \%$ of cases [1]. The standard treatment for primary thyroid cancer is surgery and radioactive iodine (RAI) ablation followed by hormonal therapy [2]. DTC has an excellent prognosis and low mortality, with disease-specific survival of up to $90 \%$ at 10-year follow-up [3]. However, the recurrence rate in the neck ranges from $20 \%$ to $59 \%$, varying according to the risk of recurrence [4]. For recurrent cancers, the current guidelines recommend reoperation followed by RAI ablation and/or thyroid hormone therapy [3]. Although reoperation is suggested as a standard treatment, distortion of the normal neck anatomy and the formation of fibrosis may increase the incidence of complications [5].

Recent studies have suggested ultrasonography (US)-guided ablation techniques, including radiofrequency ablation (RFA), ethanol ablation, and laser ablation, as minimally invasive treatments for benign thyroid nodules and recurrent thyroid cancers $[6,7]$. There are several international

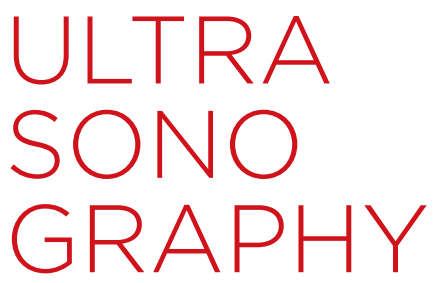

\section{REVIEW ARTICLE}

https://doi.org/10.14366/usg.21221 pISSN: 2288-5919 - elSSN: 2288-5943 Ultrasonography 2022;41:434-443

Received: October 22, 2021

Revised: December 21, 2021

Accepted: December 21, 2021

Correspondence to: Jung Hwan Baek, MD, PhD, Department of Radiology and Research Institute of Radiology, University of Ulsan College of Medicine, Asan Medical Center, 88 Olympic-ro 43-gil, Songpa-gu, Seoul 05505, Korea

Tel. +82-2-3010-4348

Fax. +82-2-476-0090

E-mail: radbaek@naver.com

This is an Open Access article distributed under the terms of the Creative Commons Attribution NonCommercial License (http://creativecommons.org/ licenses/by-nc/4.0/) which permits unrestricted noncommercial use, distribution, and reproduction in any medium, provided the original work is properly cited.

Copyright (C) 2022 Korean Society of Ultrasound in Medicine (KSUM)

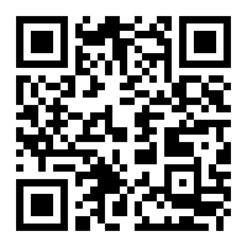

How to cite this article:

Lee MK, Baek JH, Chung SR, Choi YJ, Lee JH, Jung SL. Radiofrequency ablation of recurrent thyroid cancers: anatomy-based management. Ultrasonography. 2022 Jul;41(3):434-443. 
guidelines for the application of RFA to the thyroid. In 2012 and 2018, the Korean Society of Thyroid Radiology (KSThR) proposed thyroid RFA guidelines covering a wide range of aspects of the treatment of benign thyroid nodules and thyroid malignancies [8-10], while other international societies have also published guidelines for the thermal ablation of benign thyroid nodules [1114]. Furthermore, recent studies have reviewed these international guidelines for RFA of benign thyroid nodules $[15,16]$. However, only the KSThR has proposed guidelines for RFA of recurrent thyroid cancer [8-10], while the Italian Scientific Society, European Thyroid Association, and Cardiovascular and Interventional Radiological Society of Europe have only mentioned recommendations for RFA of recurrent thyroid cancer $[11,17]$. The KSThR recommends RFA for curative or palliative purposes in patients with high surgical risk or those who refuse repeated surgery [9]. Previous studies have reviewed the basic ablation technique, pre-procedural evaluation, efficacy, and complications of RFA for recurrent thyroid cancer $[7,18]$, and RFA has achieved therapeutic success of $95.1 \%$ and $99.5 \%$ in short-term and long-term studies, respectively $[6,19]$. Although many studies report the basic concepts and efficacy of RFA for recurrent thyroid cancers, there is a lack of detailed technical information on improving its efficacy and minimizing complications. In particular, although recurrent thyroid cancer can involve critical structures such as the trachea and skin, no studies have suggested techniques and treatment strategies for RFA of recurrent thyroid cancer according to anatomical considerations.

Therefore, in this review, we introduce anatomy-based technical aspects of RFA for recurrent thyroid cancer involving specific locations such as the trachea, esophagus, or skin, and the combination of treatments for achieving clinical success. We also briefly review the literature available on RFA of recurrent thyroid cancer, including the indications, basic techniques, clinical outcomes, and complications.

\section{Indications}

The KSThR guidelines [9] suggest that RFA has a role in the treatment of recurrent thyroid cancer in patients with high surgical risk and in those who wish to avoid repeated surgery. The guideline recommends two types of treatment strategies: curative (so-called complete) ablation and palliative management [9]. Curative ablation is defined as total ablation of any recurrent cancers visualized on US $[7,18]$. Several studies recommend performing curative ablation for recurrent thyroid cancers when three or fewer recurrent lesions are visible on neck US and there is no metastatic tumor beyond the neck at the time of treatment $[6,20-22]$. Palliative management is performed to treat recurrent thyroid cancer causing cosmetic and/ or symptomatic problems $[7,18]$, or recurrent thyroid cancer with impending invasion of critical structures such as the trachea, skin, esophagus, nerves, and vessels. There is no size limit for treating recurrent thyroid cancer, and large recurrent cancers or those invading critical structures can be further treated using combination therapies, including external beam radiation therapy (RT) $[3,23]$ and a tyrosine protein kinase inhibitor (TKI) [24-26].

\section{Pre-procedural Evaluation}

\section{US Evaluation}

Pre-procedural surveillance of recurrent thyroid cancer and adjacent critical structures is important for effective and safe ablation. Three orthogonal diameters and volumes of recurrence cancer might be assessed to provide a baseline set of measurements before RFA. Unlike the case for most benign tumors, the safety margin around recurrent thyroid cancers is generally insufficient, and procedurerelated complications are therefore more frequent $[27,28]$. Careful evaluation of critical structures on US may minimize the incidence of complications after RFA. The recurrent laryngeal nerve is the most commonly affected critical structure during RFA of recurrent thyroid cancer located in the central neck [27]. The vagus nerve is located within the carotid sheath and usually passes posterolaterally to the common carotid artery $[29,30]$, although it shows anatomical variations including anterior, medial, and posterior types located close to the thyroid bed, which can increase the risk of complications during RFA $[29,30]$. Other critical structures, including the spinal accessory nerve, cervical sympathetic ganglion, phrenic nerve, trachea, esophagus, skin, and vessels, should also be carefully evaluated before RFA of recurrent thyroid cancer, and a detailed understanding of their anatomical locations is recommended [30]. After neck dissection, traumatic neuroma may be easily confused with recurrent thyroid cancer, and its prevalence is reported to be $1.1 \%-17.8 \%$ [31-34]. Confusion between recurrent thyroid cancer and traumatic neuroma can cause unnecessary biopsy, surgery, and patient anxiety. Ablation of traumatic neuroma can induce persistent palsy of the associated nerve. Therefore, it is important to carefully evaluate the course of nerve structures and to distinguish between recurrent cancer and traumatic neuroma through a detailed US evaluation [31,32].

Various critical structures, especially nerves, are located in the central and lateral neck space, and recurrent thyroid cancer can occur anywhere within the neck space. Therefore, it is necessary to understand the anatomical location of nerves and to identify critical structures on US, while paying attention to prevent confusion between recurrent thyroid cancer and traumatic neuroma. 


\section{Computed Tomography Evaluation}

The current guidelines recommend US for the primary evaluation of recurrent thyroid cancer $[35,36]$. However, although US provides excellent diagnostic performance, it has limitations in the evaluation of deeply located and/or retropharyngeal or mediastinal lymph nodes $[37,38]$. Computed tomography (CT) can provide added value over US, improving diagnostic performance compared with US alone and providing more detailed anatomical information on recurrent thyroid cancer [39]. Therefore, CT can be helpful in treatment planning before RFA of recurrent thyroid cancer. The KSThR guidelines recommend thyroid-dedicated CT before RFA [9], and the recommended thyroid-dedicated CT protocol is posted on the KSThR website (https://www.thyroidimaging.kr/guidelines/ file/ct_2021_final.pdf). This recommended CT protocol involves early arterial-phase contrast-enhanced CT (25-40 second scan delay) with thin sections $(2-2.5 \mathrm{~mm})$. Park et al. [40] showed that a 25-second delayed image improved diagnostic accuracy for the detection of lateral cervical lymph node metastasis. The early arterial enhancement of metastatic lymph nodes is explained by tumor angiogenesis and recruitment of capsular vessels; therefore, early arterial-phase $\mathrm{CT}$ can differentiate between metastatic lymph nodes and benign lymph nodes [41]. Moreover, recent studies, including a meta-analysis, showed that thyroid CT with early arterial-phase images has good diagnostic performance in the evaluation of recurrent thyroid cancer in routine clinical practice $[39,42]$.

CT evaluation can be effective for detecting recurrent thyroid cancer that is not covered in US evaluations, and provides more detailed information on critical structures near the recurrent thyroid cancer. For effective and safe RFA of recurrent thyroid cancer, CT evaluation is an indispensable part of the pre-procedural evaluation.

\section{Technique}

\section{The Basic Techniques and Devices}

The RFA guidelines recommend the following three standard techniques for recurrent cancers [9]: perilesional lidocaine injection, hydrodissection, and the moving shot technique [9].

To control pain during RFA, the current guidelines recommend local anesthesia rather than general anesthesia or sedation $[9,11,13,14]$, with peritumoral lidocaine injection being sufficient for pain control (Fig. 1A) $[7,18]$. The moving shot technique is the standard method for the treatment of recurrent thyroid cancers $[9,28]$. This technique involves the unit-by-unit ablation of recurrent thyroid cancer, which is obtained by moving the electrode $[7,9,28]$. If the recurrent thyroid cancer is small (less than $5 \mathrm{~mm}$ ), the electrode may be fixed in the center of the lesion and not moved during the ablation $[7,18]$. Recurrent thyroid cancer is usually located near critical structures, and careful anatomical evaluation of the recurrent thyroid cancer and adjacent structures is therefore needed for safe ablation [9]. The hydrodissection technique is mandatory to prevent thermal injury to critical structures, especially when recurrent thyroid cancers are located adjacent to critical structures. Injection of $0^{\circ} \mathrm{C}-4^{\circ} \mathrm{C} 5 \%$ dextrose water (DW) with iso-osmolarity and a nonionic composition can induce separation of recurrent thyroid cancer from adjacent critical structures and play a role as a thermal barrier (Fig. 1B) $[28,43]$.

The modified straight internally-cooled electrodes that have recently been developed in Korea are shorter $(7 \mathrm{~cm})$ and thinner

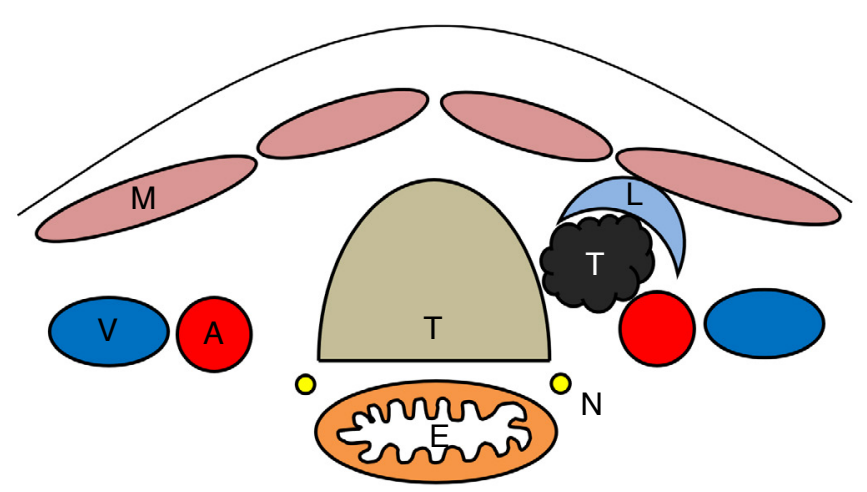

A

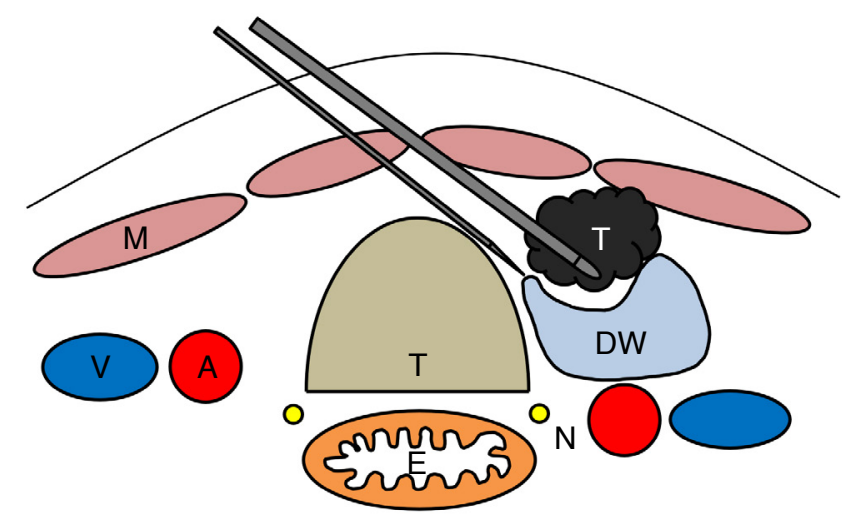

B

Fig. 1. The basic radiofrequency ablation techniques for recurrent thyroid cancer.

A. Schematic illustration shows a perilesional lidocaine injection. Lidocaine $(L)$ is injected into the superficial soft tissue around the tumor

(T) for pain control. To reduce nerve palsy due to the effects of lidocaine, a small amount of lidocaine is applied near the nerve structures. B. Schematic illustration shows the hydrodissection technique. Dextrose water is injected between the recurrent tumor (T) and critical structures. A, common carotid artery; $E$, esophagus; $M$, strap muscles; $N$, recurrent laryngeal nerve; $T$, trachea; $V$, internal jugular vein. 
(18-19 G) than conventional electrodes, and are routinely used for RFA $[9,28]$. A thin electrode of $19 \mathrm{G}$ can easily approach small recurrent thyroid cancer and reduce soft tissue thermal injury near recurrent thyroid cancer [6]. Active tips have various sizes (0.38, $0.5,0.7$, or $1.0 \mathrm{~cm})$, and operators can choose an electrode tip size according to the size of the tumor and its relationship with surrounding critical structures [7]. Table 1 lists the starting ablation power according to active tip size based on our experience $[7,44]$. The ablation zone should have a 1-2 mm safety margin to minimize marginal recurrence, especially when recurrent cancers have a spiculated margin. The ablation of recurrent thyroid cancer should be terminated when all conceptual units change to transient hyperechoic zones [9].

\section{Recurrent Thyroid Cancer Involving the Trachea and Skin}

Tracheal involvement usually occurs in the operation bed, and may increase the mortality rate due to airway obstruction [45]. For the treatment of recurrent cancer involving the trachea, anatomical considerations should be precedent (Fig. 2). The anterior and lateral walls of the trachea consist of 20 incomplete rings of hyaline cartilage, while the posterior wall consists of smooth muscles [46]. The cartilage rings are connected by annular ligaments consisting of dense fibrous tissue [47], whereas perichondral tissue consists of loose connective tissue composed of a collagenous and elastin protein-rich matrix [48]. Therefore, recurrent cancer

Table 1. RF power according to active tip size

\begin{tabular}{cc}
\hline RF power $(\mathrm{W})$ & Active tip size $(\mathrm{cm})$ \\
\hline $5-15$ & 0.38 \\
$10-30$ & 0.5 \\
$20-40$ & 0.7 \\
$50-80$ & 1.0 \\
\hline
\end{tabular}

$\mathrm{RF}$, radiofrequency.

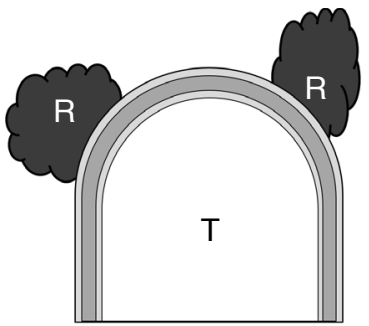

Tracheal wall involvement

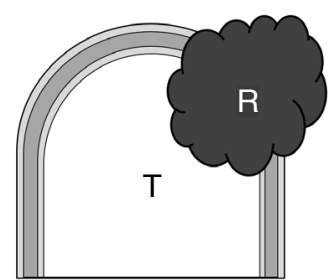

Intraluminal involvement A

Fig. 3. Recurrent thyroid cancer involving the trachea.

A. Schematic illustration of recurrent thyroid cancer $(R)$ with extratracheal or intratracheal involvement is shown. $B$. Tracheal stent pushes out the recurrent thyroid cancer with intratracheal involvement. S, stent; $T$, trachea. 
Fig. 1).

The treatment of recurrent cancer with skin involvement is challenging. Although skin involvement by recurrent thyroid cancer is not common [52-54], tumors can induce skin necrosis and blood oozing, causing poor quality of life. Therefore, we recommend early detection of skin involvement by recurrent thyroid cancer and management to prevent skin necrosis. Skin burns are the most common complication during RFA of tumors involving the skin [55]. For effective and safe RFA of such cancers, an understanding of the skin anatomy is essential. The skin is composed of three layers: the epidermis, dermis, and hypodermis (the so-called subcutaneous layer) $[56,57]$. In an anatomical evaluation on US, the echogenicity of the skin layers is divided according to tissue composition, with the keratin-rich epidermis showing as a hyperechoic line, the dermis consisting of collagen appearing as a hyperechoic band, and the hypodermis containing fat lobules presenting as a hypoechoic layer (Fig. 4) [58]. The hydrodissection technique is effective for preventing skin burns $[55,59]$, while the application of an ice bag at the ablation site can additionally reduce this complication. The risk of skin burns and the effectiveness of hydrodissection during RFA differ according to the location and composition of the skin layers. When recurrent thyroid cancer involves the epidermis, hydrodissection is less effective because of the keratin-rich compact nature of the epidermis [58]. By contrast, hypodermis with tumor involvement can be easily separated from the dermis because the hypodermis is mainly composed of loose fat lobules [57]. The main problem for the RFA treatment strategy is tumors located in the dermis, which is composed of dense collagen and elastin [58]. Ablation of recurrent thyroid cancer involving the dermal layer therefore requires careful monitoring and early treatment.

Early-stage tumors (separated from the trachea or skin by hydrodissection) can be easily treated by RFA. Therefore, early detection and treatment of tumors with tracheal or skin involvement are recommended.

\section{Post-procedural Evaluation}

As in the pre-procedural US evaluation, follow-up US evaluates the tumor size, volume reduction rate (VRR; [(initial volume-final volume $\times 100 /$ initial volume]), vascularity, and the development of new recurrent thyroid cancer or metastatic lymph nodes [9]. After RFA, the ablated tumor generally shows hyperechogenicity and decreased vascularity on color Doppler imaging. The volume of the ablated tumor may increase immediately after RFA due to edematous change and the large ablated zone over the tumor size, including the safety margin. After this, the volume gradually decreases and shows a large decline over 1-3 months $[19,60]$. Size reduction and an absence of vascularity on US are key findings indicating well-treated cancer. The duration of follow-up US and clinical evaluation are not currently clearly defined but should be decided on the basis of the treatment status and further treatment plan $[6,19,60]$. Although grayscale US and color Doppler US are primarily used to evaluate residual viable tumor portions, some studies suggest the value of contrast-enhanced US after ablation [61-63]. These studies demonstrated that contrast-enhanced US, which reflects microvascularization of tumor, is valuable for postprocedural evaluation after US-guided ablation, and reported that it has good performance for detecting recurrence at the treated tumor margin [61-63].

Thyroid-dedicated CT is also recommended for post-procedural evaluation $[9,10]$. A well-treated tumor shows a size reduction and an absence of enhancement, and CT is therefore essential for

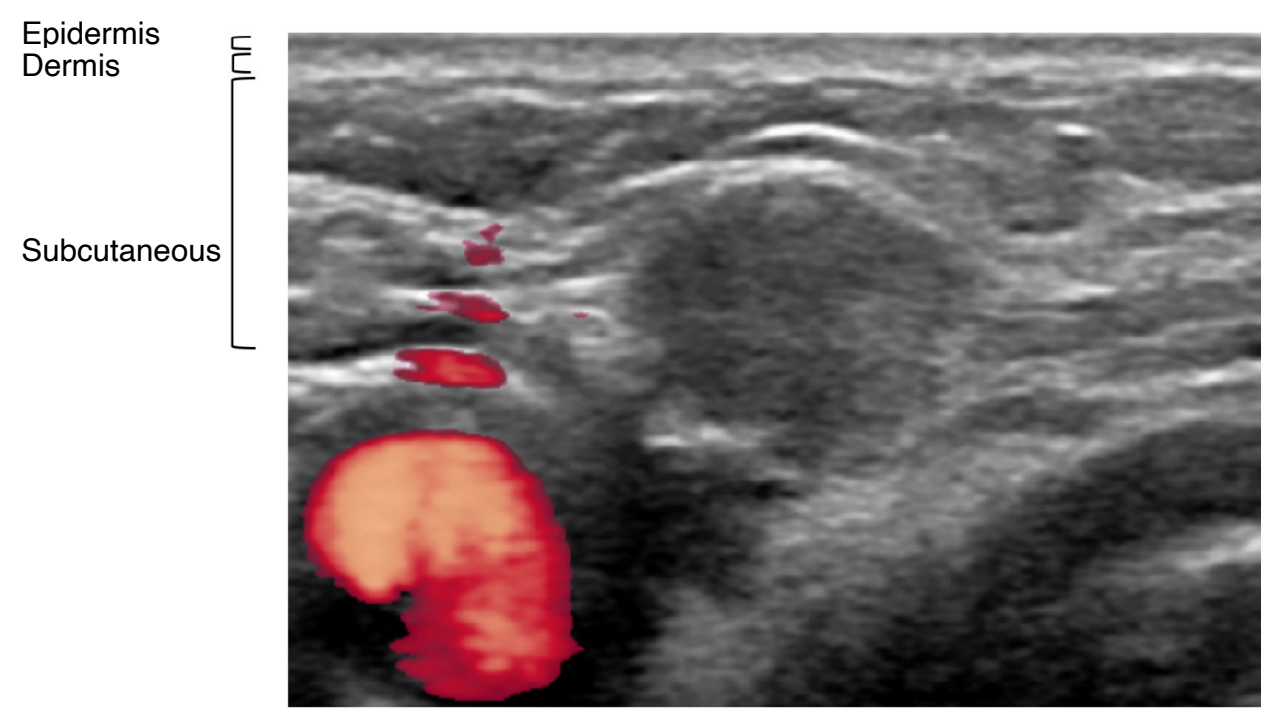

Fig. 4. Ultrasonography (US) findings of skin layers. Doppler US shows recurrent thyroid cancer involving the subcutaneous layer of the skin. 
evaluation of treatment efficacy. Serum thyroglobulin $(\mathrm{Tg})$ levels are also routinely evaluated during the follow-up period because a reduction in serum Tg levels is important for assessing treatment efficacy after RFA $[6,19]$.

The relief of tumor-related symptoms is important for evaluating treatment efficacy, especially tumors that are large or involve critical structures. Because evaluating the compromise of critical structures is difficult, tumor-related symptom relief may reflect the effectiveness of ablation.

During follow-up, evaluation of treatment efficacy is important and helpful for making further treatment plans. Treatment efficacy is usually evaluated by VRR, the therapeutic success rate (VRR $>50 \%$ ), complete disappearance of the recurrent cancer on US and/ or $\mathrm{CT}$, reduction of the serum Tg level, and relief of tumor-related symptoms $[6,9,19]$. Additional treatment is recommended if there is the presence of a power Doppler signal on follow-up US or an enhancing portion of the treated recurrent cancer on follow-up CT.

\section{Clinical Outcomes}

Many studies, including a meta-analysis, have revealed good clinical outcomes for RFA of recurrent thyroid cancers $[4,6,19,64,65]$. The general concept of the clinical outcome is well established in terms of curative treatment for recurrent thyroid cancer, but the clinical outcomes for palliative management of recurrent thyroid cancer are more complicated. The palliative management of tumors involving critical structures is more conservative because complete disappearance of tumors is difficult to achieve and tumors usually need combination therapy.

In studies evaluating curative treatment, short-term follow-up $[6,20,21,66,67]$ revealed therapeutic success rates of $75 \%$ to $95.1 \%$ and complete disappearance rates of $25 \%$ to $93.9 \%$. Although the mean follow-up period in these studies was short (range, 10.3 to 40.7 months), the studies showed good clinical outcomes. A recent meta-analysis that included 10 studies with a total of 270 patients (415 thyroid nodules) also supports these results; the therapeutic success rate was $100 \%$, complete disappearance was noted in $68.8 \%$, and a reduction in the serum $\mathrm{Tg}$ level was noted in $71.6 \%$ of cases [4]. A long-term follow-up study showed more impressive results for clinical outcomes of RFA of recurrent thyroid cancer, with therapeutic success of $99.5 \%$ and complete disappearance noted in $91.3 \%$ of cases [19]. The tumor volume typically gradually decreases after ablation, and further long-term studies are therefore likely to show better clinical outcomes for recurrent thyroid cancer. Studies using propensity score matching to compare RFA and surgery for the treatment of recurrent thyroid cancer $[64,65]$ indicated that RFA may be an effective and safe alternative treatment to surgery,
Table 2. Studies comparing RFA and surgery

\begin{tabular}{cccc}
\hline Component & Sources & RFA (\%) & Surgery (\%) \\
\hline 1-Year RFSR & Kim et al. (2015) [65] & 96.0 & 92.2 \\
& Choi et al. (2019) [64] & NA & NA \\
3-Year RFSR & Kim et al. (2015) [65] & 92.6 & 92.2 \\
& Choi et al. (2019) [64] & 100 & 97.9 \\
6-Year RFSR & Kim et al. (2015) [65] & NA & NA \\
& Choi et al. (2019) [64] & 100 & 97.8 \\
\hline
\end{tabular}

RFA, radiofrequency ablation; RFSR, recurrence-free survival rate; NA, not applicable.

with higher recurrence-free survival rates (Table 2). Although these results provide evidence supporting RFA as an alternative treatment for recurrent thyroid cancer, the tumors included in previous studies were usually located in the central and/or lateral neck, and the clinical outcomes shown are generally for curative treatment of recurrent thyroid cancer. There is a lack of studies dealing with the clinical outcomes of palliative management of large tumors or tumors involving critical structures such as the trachea and skin. Therefore, further clinical outcome studies of such tumors are necessary, and they may help in making decisions on the treatment of recurrent thyroid cancer.

\section{Complications}

The most frequent complications following RFA are discomfort and pain, but these usually resolve within several minutes to hours [68]. Pain is sometimes felt as radiating pain to the teeth, jaw, head, and chest [68]. Local pain may be reduced by injection of lidocaine, and the operator should therefore evaluate the patient's symptoms during RFA and apply lidocaine when patients complain of local pain [28].

Various complications have been reported following RFA, including pain, hemorrhage, nerve injury, and skin burns $[6,9,69]$. Major and minor complications, as well as side effects, are classified by the guidelines of the Society of Interventional Radiology (Supplementary Table 1) $[68,70]$. In patients undergoing RFA for recurrent cancers, the prevalence of total complications is reported to be $7.8 \%-10.9 \%$, whereas the prevalence of major complications is reported to be $5.4 \%-6.7 \%[27,68]$.

Voice change is the most common major complication following ablation of recurrent cancer, with an incidence of $0.8 \%-8.0 \%$ in previous studies and a meta-analysis $[27,43,68,69]$. As recurrent cancers are smaller and have no safety margins between normal thyroid tissue $[27,28]$, the rate of voice change is higher than that for the treatment of benign thyroid nodules [68]. Voice change is usually induced by injury to the recurrent laryngeal and vagus nerves 
$[27,68]$. The vagus nerve is usually detected on the US evaluation [29]. Therefore, it is possible to ovoid vagus nerve injury during RFA. However, detecting the recurrent laryngeal nerve on US, usually located in the tracheoesophageal groove, is difficult due to its small size [29]. To minimize voice change, an anatomic evaluation of nerve structures and careful control of the electrode tip are recommended for recurrent tumors near nerves, while hydrodissection techniques are effective for reducing thermal injury to nerve structures [28]. In particular, the ablation of recurrent cancer located in the tracheoesophageal groove can induce recurrent laryngeal nerve injury, so frequent checking for voice change is necessary during ablation. A recent retrospective study suggested that a cold 5\% DW injection can manage thermal nerve damage during RFA [43].

Other complications induced by nerve injury have been reported for RFA of benign thyroid nodules, such as Horner syndrome; however, no such event has been reported for RFA of recurrent cancer $[27,43,68,69]$. The symptoms of Horner syndrome are conjunctival injection, ptosis, miosis, and anhidrosis $[9,68]$, and it may be caused by thermal injury to the middle cervical sympathetic ganglion. Furthermore, spinal accessory nerve injury, which presents with limitations to shoulder movement, can be induced during RFA for recurrent thyroid cancer [68]. A detailed US evaluation of the neck is necessary to minimize nerve injury, and regular checking of symptoms may help to manage nerve injury. Skin burns are also a potential complication during RFA, especially in recurrent cancer involving the skin. The hydrodissection technique can reduce the incidence of skin burns, and the application of an ice bag is effective to relieve skin burns $[7,18]$.

\section{Conclusion}

US-guided RFA is an alternative treatment for recurrent thyroid cancer, with good outcomes and low rates of complications. A thorough understanding of neck anatomy and the RFA procedure are useful for the effective treatment of patients with high surgical risk or those who refuse repeated surgery.

ORCID: Min Kyoung Lee: https://orcid.org/0000-0003-3172-3159; Jung Hwan Baek: https://orcid.org/0000-0003-0480-4754; Sae Rom Chung: https://orcid.org/00000003-4219-7166; Young Jun Choi: https://orcid.org/0000-0002-1481-3200; Jeong Hyun Lee: https://orcid.org/0000-0002-0021-4477; So Lyung Jung: https://orcid.org/00000002-3267-8399

\section{Author Contributions}

Conceptualization: Lee MK, Baek JH, Chung SR, Choi YJ, Lee JH, Jung SL. Data acquisition: Lee MK. Data analysis or interpretation: Lee MK. Drafting of the manuscript: Lee MK. Critical revision of the manuscript: Lee MK, Baek JH, Chung SR, Choi YJ, Lee JH, Jung SL. Approval of the final version of the manuscript: all authors.

\section{Conflict of Interest}

No potential conflicts of interest relevant to this article are reported.

\section{Supplementary Material}

Supplementary Table 1. Classification of complications and side effects according to the guidelines of the Society of Interventional Radiology (https://doi.org/10.14366/usg.21221).

Supplementary Fig. 1. Treatment algorithm for the recurrent thyroid cancer involving the trachea. RFA, radiofrequency ablation; RT, radiotherapy (https://doi.org/10.14366/usg.21221).

\section{References}

1. Sherman SI. Thyroid carcinoma. Lancet 2003;361:501-511.

2. Cabanillas ME, McFadden DG, Durante C. Thyroid cancer. Lancet 2016;388:2783-2795.

3. Haugen BR. 2015 American Thyroid Association management guidelines for adult patients with thyroid nodules and differentiated thyroid cancer: what is new and what has changed? Cancer 2017;123:372-381.

4. Suh CH, Baek JH, Choi YJ, Lee JH. Efficacy and safety of radiofrequency and ethanol ablation for treating locally recurrent thyroid cancer: a systematic review and meta-analysis. Thyroid 2016;26:420-428.

5. Samaan NA, Schultz PN, Hickey RC, Goepfert H, Haynie TP, Johnston $D A$, et al. The results of various modalities of treatment of well differentiated thyroid carcinomas: a retrospective review of 1599 patients. J Clin Endocrinol Metab 1992;75:714-720.

6. Lim HK, Baek JH, Lee JH, Kim WB, Kim TY, Shong YK, et al. Efficacy and safety of radiofrequency ablation for treating locoregional recurrence from papillary thyroid cancer. Eur Radiol 2015;25:163170.

7. Jeong SY, Baek JH, Choi YJ, Lee JH. Ethanol and thermal ablation for malignant thyroid tumours. Int J Hyperthermia 2017;33:938945.

8. Kim JH, Baek JH, Lim HK, Na DG. Summary of the 2017 thyroid radiofrequency ablation guideline and comparison with the 2012 guideline. Ultrasonography 2019;38:125-134.

9. Kim JH, Baek JH, Lim HK, Ahn HS, Baek SM, Choi YJ, et al. 2017 Thyroid radiofrequency ablation guideline: Korean Society of Thyroid Radiology. Korean J Radiol 2018;19:632-655.

10. Na DG, Lee JH, Jung SL, Kim JH, Sung JY, Shin JH, et al. Radiofrequency ablation of benign thyroid nodules and recurrent thyroid cancers: consensus statement and recommendations. 
Korean J Radiol 2012;13:117-125.

11. Garberoglio R, Aliberti C, Appetecchia M, Attard M, Boccuzzi G, Boraso $F$, et al. Radiofrequency ablation for thyroid nodules: which indications? The first Italian opinion statement. J Ultrasound 2015;18:423-430.

12. National Institute for Health and Care Excellence. Ultrasoundguided percutaneous radiogrequency ablation for benign thyroid nodules: interventional procedure guidance [Internet]. London: National Institute for Health and Care Excellence, 2016 [cited 2016 Jun 22]. Available from: https://www.nice.org.uk/guidance/ipg562.

13. Dobnig H, Zechmann W, Hermann M, Lehner M, Heute D, Mirzaei $S$, et al. Radiofrequency ablation of thyroid nodules: "Good Clinical Practice Recommendations" for Austria: an interdisciplinary statement from the following professional associations: Austrian Thyroid Association (OSDG), Austrian Society for Nuclear Medicine and Molecular Imaging (OGNMB), Austrian Society for Endocrinology and Metabolism (OGES), Surgical Endocrinology Working Group (ACE) of the Austrian Surgical Society (OEGCH). Wien Med Wochenschr 2020;170:6-14.

14. Papini E, Pacella CM, Solbiati LA, Achille G, Barbaro D, Bernardi $S$, et al. Minimally-invasive treatments for benign thyroid nodules: a Delphi-based consensus statement from the Italian minimallyinvasive treatments of the thyroid (MITT) group. Int J Hyperthermia 2019;36:376-382.

15. Lee $M$, Baek JH, Suh $\mathrm{CH}$, Chung SR, Choi YJ, Lee JH, et al. Clinical practice guidelines for radiofrequency ablation of benign thyroid nodules: a systematic review. Ultrasonography 2021;40:256-264.

16. Ha EJ, Baek JH, Che Y, Chou YH, Fukunari N, Kim JH, et al. Radiofrequency ablation of benign thyroid nodules: recommendations from the Asian Conference on Tumor Ablation Task Force. Ultrasonography 2021:40:75-82.

17. Mauri G, Hegedus L, Bandula S, Cazzato RL, Czarniecka A, Dudeck 0 , et al. European Thyroid Association and Cardiovascular and Interventional Radiological Society of Europe 2021 Clinical practice guideline for the use of minimally invasive treatments in malignant thyroid lesions. Eur Thyroid J 2021;10:185-197.

18. Shin JE, Baek JH, Lee JH. Radiofrequency and ethanol ablation for the treatment of recurrent thyroid cancers: current status and challenges. Curr Opin Oncol 2013;25:14-19.

19. Chung SR, Baek JH, Choi YJ, Lee JH. Longer-term outcomes of radiofrequency ablation for locally recurrent papillary thyroid cancer. Eur Radiol 2019;29:4897-4903.

20. Baek JH, Kim YS, Sung JY, Choi H, Lee JH. Locoregional control of metastatic well-differentiated thyroid cancer by ultrasoundguided radiofrequency ablation. AJR Am J Roentgenol 2011;197:W331-W336.

21. Lee SJ, Jung SL, Kim BS, Ahn KJ, Choi HS, Lim DJ, et al. Radiofrequency ablation to treat loco-regional recurrence of welldifferentiated thyroid carcinoma. Korean J Radiol 2014;15:817-
826.

22. Hay ID, Lee RA, Davidge-Pitts C, Reading CC, Charboneau JW. Longterm outcome of ultrasound-guided percutaneous ethanol ablation of selected "recurrent" neck nodal metastases in 25 patients with TNM stages III or IVA papillary thyroid carcinoma previously treated by surgery and 131I therapy. Surgery 2013;154:1448-1454.

23. Jacomina LE, Jacinto JK, Co LB, Yu KK, Agas RA, Co JL, et al. The role of postoperative external beam radiotherapy for differentiated thyroid carcinoma: a systematic review and meta-analysis. Head Neck 2020;42:2181-2193.

24. Bonichon F, Buy X, Godbert Y, Pointillart V, Henriques de Figueiredo $B$, Gangi $A$, et al. Local treatment of metastases from differentiated thyroid cancer. Ann Endocrinol (Paris) 2015;76:1S40-1S46.

25. Valerio L, Pieruzzi L, Giani C, Agate L, Bottici V, Lorusso L, et al. Targeted therapy in thyroid cancer: state of the art. Clin Oncol ( $R$ Coll Radiol) 2017;29:316-324.

26. Fleeman $N$, Houten $R$, Chaplin $M$, Beale S, Boland A, Dundar $Y$, et al. A systematic review of lenvatinib and sorafenib for treating progressive, locally advanced or metastatic, differentiated thyroid cancer after treatment with radioactive iodine. BMC Cancer 2019;19:1209.

27. Chung SR, Suh CH, Baek JH, Park HS, Choi YJ, Lee JH. Safety of radiofrequency ablation of benign thyroid nodules and recurrent thyroid cancers: a systematic review and meta-analysis. Int J Hyperthermia 2017;33:920-930.

28. Park HS, Baek JH, Park AW, Chung SR, Choi YJ, Lee JH. Thyroid radiofrequency ablation: updates on innovative devices and techniques. Korean J Radiol 2017;18:615-623.

29. Ha EJ, Baek JH, Lee JH, Kim JK, Shong YK. Clinical significance of vagus nerve variation in radiofrequency ablation of thyroid nodules. Eur Radiol 2011;21:2151-2157.

30. Ha EJ, Baek JH, Lee JH. Ultrasonography-based thyroidal and perithyroidal anatomy and its clinical significance. Korean J Radiol 2015;16:749-766.

31. Yabuuchi H, Kuroiwa T, Fukuya T, Tomita K, Hachitanda Y. Traumatic neuroma and recurrent lymphadenopathy after neck dissection: comparison of radiologic features. Radiology 2004;233:523-529.

32. Kwak JY, Kim EK, Kim MJ, Son E. Sonographic features of traumatic neuromas after neck dissection. J Clin Ultrasound 2009;37:189 193.

33. Huang LF, Weissman JL, Fan C. Traumatic neuroma after neck dissection: CT characteristics in four cases. AJNR Am J Neuroradiol 2000;21:1676-1680.

34. Ha EJ, Baek JH, Lee JH, Kim YJ, Kim JK, Kim TY, et al. Characteristic ultrasound feature of traumatic neuromas after neck dissection: direct continuity with the cervical plexus. Thyroid 2012;22:820-826.

35. Gharib H, Papini E, Paschke R, Duick DS, Valcavi R, Hegedus L, et al. American Association of Clinical Endocrinologists, Associazione Medici Endocrinologi, and EuropeanThyroid Association Medical 
guidelines for clinical practice for the diagnosis and management of thyroid nodules. Endocr Pract 2010;16 Suppl 1:1-43.

36. Shin JH, Baek JH, Chung J, Ha EJ, Kim JH, Lee YH, et al. Ultrasonography diagnosis and imaging-based management of thyroid nodules: revised Korean Society of Thyroid Radiology consensus statement and recommendations. Korean J Radiol 2016;17:370-395.

37. Mulla M, Schulte KM. Central cervical lymph node metastases in papillary thyroid cancer: a systematic review of imaging-guided and prophylactic removal of the central compartment. Clin Endocrinol (Oxf) 2012;76:131-136.

38. Mulla MG, Knoefel WT, Gilbert J, McGregor A, Schulte KM. Lateral cervical lymph node metastases in papillary thyroid cancer: a systematic review of imaging-guided and prophylactic removal of the lateral compartment. Clin Endocrinol (Oxf) 2012;77:126-131.

39. Lee Y, Kim JH, Baek JH, Jung SL, Park SW, Kim J, et al. Value of $\mathrm{CT}$ added to ultrasonography for the diagnosis of lymph node metastasis in patients with thyroid cancer. Head Neck 2018;40:2137-2148.

40. Park JE, Lee JH, Ryu KH, Park HS, Chung MS, Kim HW, et al. Improved diagnostic accuracy using arterial phase $\mathrm{CT}$ for lateral cervical lymph node metastasis from papillary thyroid cancer. AJNR Am J Neuroradiol 2017;38:782-788.

41. Na DG, Lim HK, Byun HS, Kim HD, Ko YH, Baek JH. Differential diagnosis of cervical lymphadenopathy: usefulness of color Doppler sonography. AJR Am J Roentgenol 1997;168:1311-1316.

42. Cho SJ, Suh CH, Baek JH, Chung SR, Choi YJ, Lee JH. Diagnostic performance of $\mathrm{CT}$ in detection of metastatic cervical lymph nodes in patients with thyroid cancer: a systematic review and metaanalysis. Eur Radiol 2019;29:4635-4647.

43. Chung SR, Baek JH, Choi YJ, Lee JH. Management strategy for nerve damage during radiofrequency ablation of thyroid nodules. Int J Hyperthermia 2019;36:204-210.

44. Baek JH, Ha EJ, Choi YJ, Sung JY, Kim JK, Shong YK. Radiofrequency versus ethanol ablation for treating predominantly cystic thyroid nodules: a randomized clinical trial. Korean J Radiol 2015;16:13321340.

45. Shaha AR. Recurrent differentiated thyroid cancer. Endocr Pract 2012;18:600-603.

46. Brand-Saberi BE, Schafer T. Trachea: anatomy and physiology. Thorac Surg Clin 2014;24:1-5.

47. Shin DH, Mark EJ, Suen HC, Grillo HC. Pathologic staging of papillary carcinoma of the thyroid with airway invasion based on the anatomic manner of extension to the trachea: a clinicopathologic study based on 22 patients who underwent thyroidectomy and airway resection. Hum Pathol 1993;24:866-870.

48. Dhasmana A, Singh A, Rawal S. Biomedical grafts for tracheal tissue repairing and regeneration "Tracheal tissue engineering: an overview". J Tissue Eng Regen Med 2020;14:653-672.
49. Chung SR, Baek JH, Choi YJ, Sung TY, Song DE, Kim TY, et al. Efficacy of radiofrequency ablation for recurrent thyroid cancer invading the airways. Eur Radiol 2021;31:2153-2160.

50. Haugen BR, Alexander EK, Bible KC, Doherty GM, Mandel SJ, Nikiforov YE, et al. 2015 American Thyroid Association management guidelines for adult patients with thyroid nodules and differentiated thyroid cancer: the American Thyroid Association Guidelines Task Force on Thyroid Nodules and Differentiated Thyroid Cancer. Thyroid 2016;26:1-133.

51. Cervelli R, Mazzeo S, De Napoli L, Boccuzzi A, Pontillo-Contillo B, Materazzi $G$, et al. Radiofrequency ablation in the treatment of benign thyroid nodules: an efficient and safe alternative to surgery. J Vasc Interv Radiol 2017;28:1400-1408.

52. Soylu S, Arikan AE, Teksoz S, Ozcan M, Bukey Y. Skin metastasis on the neck: an unusual presentation of recurrence of papillary thyroid carcinoma. Gland Surg 2017;6:594-597.

53. Somoza AD, Bui H, Samaan S, Dhanda-Patil R, Mutasim DF. Cutaneous metastasis as the presenting sign of papillary thyroid carcinoma. J Cutan Pathol 2013;40:274-278.

54. Koller EA, Tourtelot JB, Pak HS, Cobb MW, Moad JC, Flynn EA. Papillary and follicular thyroid carcinoma metastatic to the skin: a case report and review of the literature. Thyroid 1998;8:10451050.

55. Guo H, Liu XL, Wang YL, Li JY, Lu WZ, Xian JZ, et al. Protection of skin with subcutaneous administration of $5 \%$ dextrose in water during superficial radiofrequency ablation in a rabbit model. Int J Hyperthermia 2014;30:258-265.

56. Losquadro WD. Anatomy of the skin and the pathogenesis of nonmelanoma skin cancer. Facial Plast Surg Clin North Am 2017;25:283-289.

57. Yousef $H$, Alhajj M, Sharma S. Anatomy, skin (integument), epidermis. Treasure Island, FL: StatPearls Publishing, 2017.

58. Barcaui Ede O, Carvalho AC, Valiante PM, Barcaui CB. Highfrequency ultrasound associated with dermoscopy in pre-operative evaluation of basal cell carcinoma. An Bras Dermatol 2014;89:828831.

59. Koo JH, Shin JH, Oh YL, Ko EY, Han BK. Sonographically guided radiofrequency ablation with and without a superficial saline injection to prevent skin burns in a rabbit model. J Ultrasound Med 2012;31:873-878.

60. Xiao J, Zhang $Y$, Zhang $M$, Lan $Y$, Yan L, Luo $Y$, et al. Ultrasonography-guided radiofrequency ablation vs. surgery for the treatment of solitary T1bNOM0 papillary thyroid carcinoma: a comparative study. Clin Endocrinol (Oxf) 2021;94:684-691.

61. Papini E, Bizzarri G, Bianchini A, Valle D, Misischi I, Guglielmi R, et al. Percutaneous ultrasound-guided laser ablation is effective for treating selected nodal metastases in papillary thyroid cancer. J Clin Endocrinol Metab 2013;98:E92-E97.

62. Zhou W, Jiang S, Zhan W, Zhou J, Xu S, Zhang L. Ultrasound-guided 
percutaneous laser ablation of unifocal T1NOM0 papillary thyroid microcarcinoma: preliminary results. Eur Radiol 2017;27:29342940.

63. Zhou W, Zhang L, Zhan W, Jiang S, Zhu Y, Xu S. Percutaneous laser ablation for treatment of locally recurrent papillary thyroid carcinoma <15 mm. Clin Radiol 2016;71:1233-1239.

64. Choi Y, Jung SL, Bae JS, Lee SH, Jung CK, Jang J, et al. Comparison of efficacy and complications between radiofrequency ablation and repeat surgery in the treatment of locally recurrent thyroid cancers: a single-center propensity score matching study. Int J Hyperthermia 2019;36:359-367.

65. Kim JH, Yoo WS, Park YJ, Park DJ, Yun TJ, Choi SH, et al. Efficacy and safety of radiofrequency ablation for treatment of locally recurrent thyroid cancers smaller than $2 \mathrm{~cm}$. Radiology 2015;276:909-918.

66. Dupuy DE, Monchik JM, Decrea C, Pisharodi L. Radiofrequency ablation of regional recurrence from well-differentiated thyroid malignancy. Surgery 2001;130:971-977.

67. Monchik JM, Donatini G, lannuccilli J, Dupuy DE. Radiofrequency ablation and percutaneous ethanol injection treatment for recurrent local and distant well-differentiated thyroid carcinoma. Ann Surg 2006;244:296-304.

68. Kim C, Lee JH, Choi YJ, Kim WB, Sung TY, Baek JH. Complications encountered in ultrasonography-guided radiofrequency ablation of benign thyroid nodules and recurrent thyroid cancers. Eur Radiol 2017;27:3128-3137.

69. Baek JH, Lee JH, Sung JY, Bae JI, Kim KT, Sim J, et al. Complications encountered in the treatment of benign thyroid nodules with USguided radiofrequency ablation: a multicenter study. Radiology 2012;262:335-342.

70. Sacks D, McClenny TE, Cardella JF, Lewis CA. Society of interventional radiology clinical practice guidelines. J Vasc Interv Radiol 2003;14(9 Pt 2):S199-S202. 